\title{
Phage vB_BveM-Goe7 represents a new genus in the subfamily Bastillevirinae
}

\author{
Alexandra Dominique Furrer ${ }^{1} \cdot$ Mechthild Bömeke $^{1} \cdot$ Michael Hoppert $^{2} \cdot$ Robert Hertel $^{1} \mathbb{C}$
}

Received: 3 September 2019 / Accepted: 8 January 2020 / Published online: 12 February 2020

(c) The Author(s) 2020

\begin{abstract}
Bacillus velezensis FZB42 is a Gram-positive, endospore-forming rhizobacterium that is associated with plant roots and promotes plant growth. It was used as host to isolate phage vB_BveM-Goe7 (Goe7). Goe7 exhibits a Myoviridae morphology with a contractile tail and an icosahedral head. Its genome is 158,674 bp in size and contains 5137-bp-long terminal repeats (LTRs). It also contains five tRNA-encoding genes and 251 coding DNA sequences (CDS), of which 65 were annotated. The adsorption constant of Goe7 is $6.1 \pm 0.24 \times 10^{-8} \mathrm{ml} / \mathrm{min}$, with a latency period of $75 \mathrm{~min}$ and a burst size of 114 particles per burst. A BLASTn sequence comparison against the non-redundant nucleotide database of NCBI revealed that Goe7 is most similar to Bacillus subtilis phage vB_BsuM-Goe3.
\end{abstract}

Bacillus velezensis FZB42 is a rod-shaped aerobic soil bacterium [1]. This member of the B. subtilis species complex [2] was originally proposed as the type strain of Bacillus amyloliquefaciens subsp. plantarum before it was recognized as B. velezensis [3]. Many strains of this group are root-associated and are able to promote plant growth [4]. We used strain FZB42 as host for the isolation of phage vB_BveM-Goe7 (Goe7) from the Göttingen municipal sewage plant (Göttingen, Germany, 51 ${ }^{\circ} 33^{\prime} 15.4^{\prime \prime} \mathrm{N} 9^{\circ} 55^{\prime} 06.4^{\prime \prime}$ E). For this, an overlay plaque assay was used. Goe7 could be isolated as a clear plaque in the bacterial layer, indicating a lytic life style. The morphology of Goe7 was determined via electron microscopy, using a Jeol 1101 electron microscope (Eching, Munich, Germany) as described previously

Handling Editor: Tim Skern.

Electronic supplementary material The online version of this article (https://doi.org/10.1007/s00705-020-04546-1) contains supplementary material, which is available to authorized users.

Robert Hertel

rhertel@gwdg.de

1 Department of Genomic and Applied Microbiology \& Göttingen Genomics Laboratory, Institute of Microbiology and Genetics, Georg-August-University Göttingen, Göttingen, Germany

2 Department of General Microbiology, Institute of Microbiology and Genetics, Georg-August-University Göttingen, Göttingen, Germany
[5]. TEM micrographs revealed a Caudovirales-related head-tail morphology (Fig. 1) and a Myoviridae-like contractile tail (Fig. 1A) [6]. A double baseplate upon tail contraction (Fig. 1A) indicates a morphological relatedness to the SPO1-like phages [7]. The icosahedral head is $88.2 \mathrm{~nm}$ $\pm 4 \mathrm{~nm}$ in diameter and the tail is $216 \mathrm{~nm} \pm 10.4 \mathrm{~nm}$ long and $16.4 \mathrm{~nm} \pm 1.9 \mathrm{~nm}$ wide.

Genome sequencing and quality assessment of the obtained sequence reads were done as described previously [5]. Initial assembly was performed with SPAdes version 3.10.1 [8]. The ends of the long terminal repeats (LTR) were resolved via Sanger sequencing as described for phage $\mathrm{vB}_{-}$ BsuM-Goe2 (Goe2) and VB_BsuM-Goe3 (Goe3) [9] using the primers Goe3_P4 (5'GCGGTATGTCTGAATAAGGG) and PP004 (5'GCACATGACAGGGATTCAAC).

The genome of Goe7 consists of a linear, double-stranded DNA chromosome with a size of 158,674 bp and a GCcontent of $41.84 \%$. Automatic annotation using the Prokka pipeline [10] identified five tRNA-encoding genes and 251 coding DNA sequence (CDS). A domain search using InterProScan [11] (Supplemental Table S1) was used to improve the initial annotation of Prokka and led to the annotation of 65 protein coding genes (Supplemental Table S2). About three quarters of the protein genes remained hypothetical, indicating hitherto unknown genes and functions.

BLASTn comparison of Goe7 with the non-redundant nucleotide database of NCBI revealed the highest similarity to Bacillus phage vB_BsuM-Goe3 [9]. It showed 96\% query coverage with $98.92 \%$ identity. Further related 

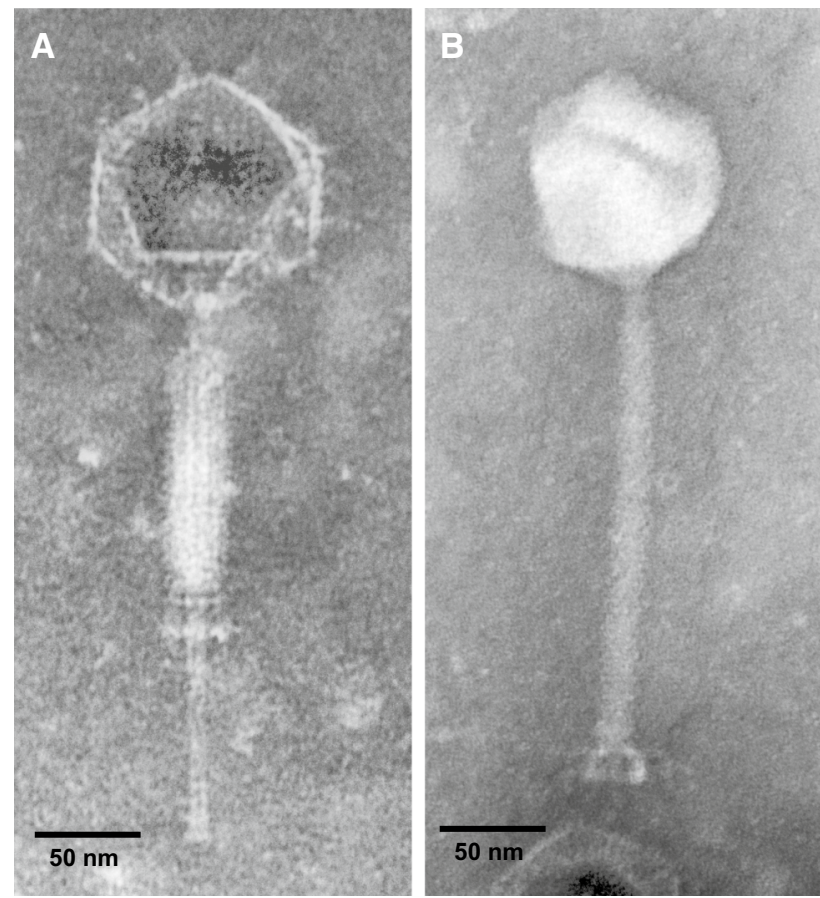

Fig. 1 vB_BveM-Goe7 micrographs. (A) Goe7 with contracted tail sheath. The baseplate reveals a double stack structure. (B) Complete infectious Goe7 particle

phages with a query coverage of $>20 \%$ were Bacillus phage BSP38 (MH606185.1) [12], SBSphiJ (LT960608.1), Grass (KF669652.1) [13] and phiNIT1 (AP013029.1), all of which are members of the lytic group of SPO1-related phages [7]. Average nucleotide identity (ANI) values [14] were calculated using the average_nucleotide_identity.py (https://githu b.com/widdowquinn/pyani) script with the ANIm option employing MUMer3 [15]. The genome sequence of Goe7 was compared with those of members of the phage family Herelleviridae, which infect members of the genus Bacillus [16]. The results revealed Goe7 to be a unique isolate, with $98.18 \%$ overall genome sequence identity to $B$. subtilis phage Goe3, indicating that it belongs to the same species (Fig. 2 and Supplemental Table S3). ANIm alignment lengths (Supplemental Table S4) perfectly reassembled all genera of the subfamilies Bastillevirinae and Spounavirinae as proposed by Barylski et al. [16]. In this context it becomes evident that Goe 7 and Goe3 form a separate genus. This new genus fits into the subfamily Bastillevirinae, as Goe7 shows similarities to members of the genera Agatevirus (Bobb $86.7 \%$ and Bp8p 87.1\%) and Nitunavirus (Grass 84.1\%, SPG24 83.7\%, phiNIT1 83.1\%) (Supplemental Table S3).

The adsorption constant of Goe7 is $6.1 \times 0.24^{-8} \mathrm{ml} / \mathrm{min}$ and was determined as described by A. M. Kropinski [17]. A latency period of 75 min and a burst size of 114 particles per burst were observed in a one-step growth experiment carried out as described by Hyman and Abedon [18] in a 250 -ml conical flask with $25 \mathrm{ml}$ of LB with vigorous shaking at $30{ }^{\circ} \mathrm{C}$ (see Table S5). Goe3, which infects B. subtilis, a close relative of $B$. velezensis, differs from Goe7 in its effect on its host [9]. Although Goe7 has an identical burst size (114 particles per burst), it adsorbs more efficiently to its host than Goe3 (adsorption constant, $8 \times 10^{-11} \mathrm{ml} / \mathrm{min}$ ) and requires more time for replication than Goe 3 latency period (55 $\mathrm{min})$.

The final genome sequence of Goe7 is publicly available in the GenBank database with the accession number MN043730. Biological samples of vB_BveM-Goe7 with the sample number DSM109177 are available in the German Collection of Microorganisms and Cell Cultures (DSMZ). 


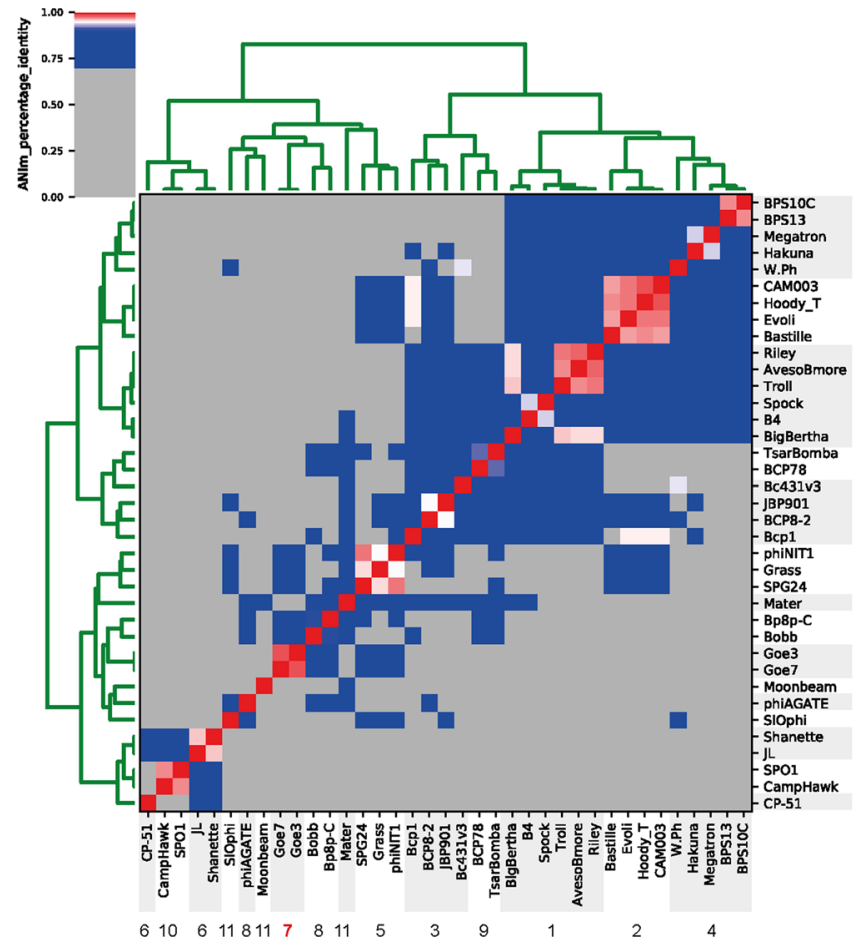

Fig. 2 Average nucleotide identity and alignment length analysis of Goe7. Genera as described by Barylski et al. [16] were used for this investigation and are indicated with shaded gray bars $(1=$ Bequatrovirus, $2=$ Bastillevirus, $3=$ Caeruleovirus, $4=$ Wphvirus, $5=$ Nitunavirus, $6=$ Siminovitchvirus, $8=$ Agatevirus, $9=$ Tsarbombavirus, $10=$ Okubovirus, $11=$ recently unassigned strains). The average nucleotide identity values were calculated with the ANIm option. Percentage identity values are visualized on the left. A reddish color

Acknowledgements Open Access funding provided by Projekt DEAL. We would like to thank Professor Dr. Rolf Daniel for scientific advice and guidance, and Dr. Anja Poehlein for sequencing. We thank Birthe Nordmann and Tobias Schilling for their help in the lab, and Avril von Hoyningen-Huene for proofreading the manuscript. This project was funded by the Volkswagen Foundation (Re. 94045).

\section{Compliance with ethical standards}

Conflict of interest The authors have no conflict of interest to declare.

Ethical approval This article does not contain any studies with human participants or animals performed by any of the authors.

Open Access This article is licensed under a Creative Commons Attribution 4.0 International License, which permits use, sharing, adaptation, distribution and reproduction in any medium or format, as long as you give appropriate credit to the original author(s) and the source, provide a link to the Creative Commons licence, and indicate if changes were made. The images or other third party material in this article are included in the article's Creative Commons licence, unless indicated otherwise in a credit line to the material. If material is not included in the article's Creative Commons licence and your intended use is not permitted by statutory regulation or exceeds the permitted use, you will

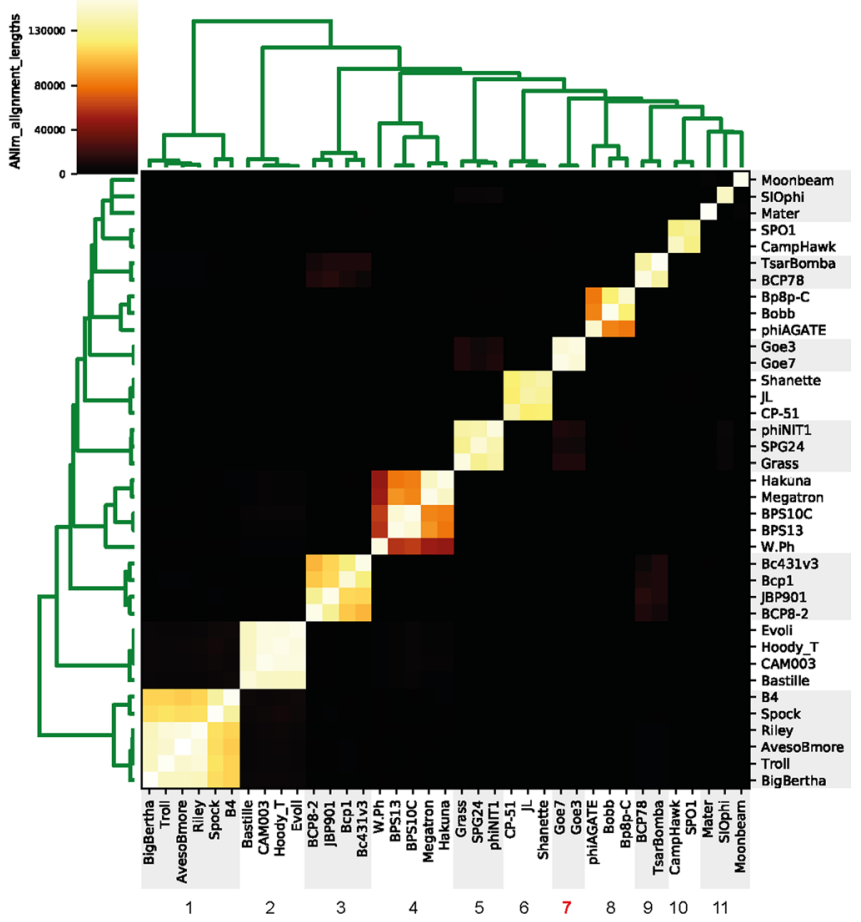

indicates the same species with an ANI value $>95 \%$. White indicates an ANI value of $\sim 95 \%$ and the species boundary. A bluish color indicates an ANI value of 70-95\% and a high degree of relatedness. Alignment lengths are visualized on the right. The brighter the color from black over red and yellow to white, the longer is the related alignment. Goe7 is of the same species as Goe3 due to $98 \%$ genome identity (left). Both strains form a new genus (7), as indicated by the alignment length of the ANIm analysis (right)

need to obtain permission directly from the copyright holder. To view a copy of this licence, visit http://creativecommons.org/licenses/by/4.0/.

\section{References}

1. Chen XH, Koumoutsi A, Scholz R, Eisenreich A, Schneider K, Heinemeyer I, Morgenstern B, Voss B, Hess WR, Reva O et al (2007) Comparative analysis of the complete genome sequence of the plant growth-promoting bacterium Bacillus amyloliquefaciens FZB42. Nat Biotechnol 25:1007-1014

2. Fan B, Blom J, Klenk HP, Borriss R (2017) Bacillus amyloliquefaciens, Bacillus velezensis, and Bacillus siamensis form an "Operational Group B. amyloliquefaciens" within the B. subtilis species complex. Front Microbiol 8:1-15

3. Dunlap CA, Kim S-J, Kwon S-W, Rooney AP (2016) Bacillus velezensis is not a later heterotypic synonym of Bacillus amyloliquefaciens; Bacillus methylotrophicus, Bacillus amyloliquefaciens subsp. plantarum and 'Bacillus oryzicola' are later heterotypic synonyms of Bacillus velezensis based on phylogenom. Int J Syst Evol Microbiol 66:1212-1217

4. Fan B, Wang C, Song X, Ding X, Wu L, Wu H, Gao X, Borriss R (2018) Bacillus velezensis FZB42 in 2018: the gram-positive model strain for plant growth promotion and biocontrol. Front Microbiol 9:1-14 
5. Nordmann B, Schilling T, Hoppert M, Hertel R (2019) Complete genome sequence of the virus isolate vB_BthM-Goe5 infecting Bacillus thuringiensis. Arch Virol 164:1485-1488

6. Ackermann H-W, Prangishvili D (2012) Prokaryote viruses studied by electron microscopy. Arch Virol 157:1843-1849

7. Klumpp J, Lavigne R, Loessner MJ, Ackermann HW (2010) The SPO1-related bacteriophages. Arch Virol 155:1547-1561

8. Bankevich A, Nurk S, Antipov D, Gurevich AA, Dvorkin M, Kulikov AS, Lesin VM, Nikolenko SI, Pham S, Prjibelski AD et al (2012) SPAdes: a new genome assembly algorithm and its applications to single-cell sequencing. J Comput Biol 19:455-477

9. Willms IM, Hoppert M, Hertel R (2017) Characterization of Bacillus subtilis Viruses vB_BsuM-Goe2 and vB_BsuM-Goe3. Viruses 9:146

10. Seemann T (2014) Prokka: rapid prokaryotic genome annotation. Bioinformatics 30:2068-2069

11. Jones P, Binns D, Chang H-Y, Fraser M, Li W, McAnulla C, McWilliam H, Maslen J, Mitchell A, Nuka G et al (2014) InterProScan 5: genome-scale protein function classification. Bioinformatics 30:1236-1240

12. Ghosh K, Kim K-P (2019) Complete nucleotide sequence analysis of a novel Bacillus subtilis-infecting phage, BSP38, possibly belonging to a new genus in the subfamily Spounavirinae. Arch Virol 164:875-878
13. Miller SY, Colquhoun JM, Perl AL, Chamakura KR, Kuty Everett GF (2013) Complete genome of Bacillus subtilis myophage grass. Genome Announc 1:30257

14. Richter M, Rosselló-Móra R (2009) Shifting the genomic gold standard for the prokaryotic species definition. Proc Natl Acad Sci 106:19126-19131

15. Kurtz S, Phillippy A, Delcher AL, Smoot M, Shumway M, Antonescu C, Salzberg SL (2004) Versatile and open software for comparing large genomes. Genome Biol 5:R12

16. Barylski J, Enault F, Dutilh BE, Schuller MB, Edwards RA, Gillis A, Klumpp J, Knezevic P, Krupovic M, Kuhn JH et al (2020) Analysis of spounaviruses as a case study for the overdue reclassification of tailed phages. Syst Biol 69(1):110-123

17. Kropinski AM (2009) Measurement of the rate of attachment of bacteriophage to cells. Methods Mol Biol 501:151-155

18. Hyman P, Abedon ST (2009) Practical methods for determining phage growth parameters. Methods Mol Biol 501:175-202

Publisher's Note Springer Nature remains neutral with regard to jurisdictional claims in published maps and institutional affiliations. 